\title{
MICROARRAY TECHNOLOGY REVEALS POTENTIALLY NOVEL GENES AND PATHWAYS INVOLVED IN NON-FUNCTIONING PITUITARY ADENOMAS
}

\author{
Qiao $\mathrm{X}^{1}$, Wang $\mathrm{H}^{2}$, Wang $\mathrm{X}^{2}$, Zhao $\mathrm{B}^{2}$, Liu J ${ }^{2, *}$
}

*Corresponding Author: Jun Liu, M.D., Department of Neurosurgery, The Second Hospital of Jilin University, 218 Ziqiang Road, Changchun, 130021, Jilin Province, People’s Republic of China. Tel: +86-138-0431-7080. E-mail: LiuJun66@126.com

\begin{abstract}
Microarray data of non-functioning pituitary adenomas (NFPAs) were analyzed to disclose novel genes and pathways involved in NFPA tumorigenesis. Raw microarray data were downloaded from Gene Expression Omnibus. Data pre-treatment and differential analysis were conducted using packages in $R$. Functional and pathway enrichment analyses were performed using package GOstats. A protein-protein interaction (PPI) network was constructed using server STRING and Cytoscape. Known genes involved in pituitary adenomas (PAs), were obtained from the Comparative Toxicogenomics Database. A total of 604 differentially expressed genes (DEGs) were identified between NFPAs and controls, including 177 up- and 427 down-regulated genes. Jak-STAT and p53 signaling pathways were significantly enriched by DEGs. The PPI network of DEGs was constructed, containing 99 up- and 288 down-regulated known disease genes (e.g. EGFR and ESR 1) as well as 16 up- and 17 down-regulated potential novel NFPAs-related genes (e.g. COL4A5, LHX3, MSN, and GHSR). Genes like COL4A5, LHX3, MSN, and GHSR and pathways such as p53 signaling and Jak-STAT signaling, might participate in NFPA development. Although further validations are required, these findings might provide guidance for future basic and therapy researches.
\end{abstract}

\footnotetext{
${ }^{1}$ Department of Gastrointestinal Surgery, The Second Hospital of Jilin University, Changchun, Jilin Province, People's Republic of China

2 Department of Neurosurgery, The Second Hospital of Jilin University, Changchun, Jilin Province, People's Republic of China
}

Keywords: Differentially expressed genes (DEGs); Functional enrichment analysis; Microarray; Non-functioning pituitary adenomas (NFPAs); Protein-protein interaction (PPI).

\section{INTRODUCTION}

As a kind of benign adenomas in the pituitary gland, clinically non-functioning pituitary adenomas (NFPAs) are the most common type of pituitary macroadenomas in adults. The NFPAs account for about $34.0 \%$ [1] of all pituitary adenomas (PAs) that occur at a prevalence rate of 75-94 per 100,000 [1,2]. Patients with NFPAs generally suffer from headaches, hypopituitarism, hypogonadism and visual field defects. Late diagnosis due to inconspicuous signs and symptoms, extension to the cavernous sinus and sellar floor, resistance to pharmacological therapy and high recurrence rate, make their treatment disappointing and challenging [3]. Approximately $80.0 \%$ of NFPAs originate from gonadotroph cells (gonadotroph pituitary adenoma, GnPA) [4], and other NFPAs are mainly associated with null cells (null cell pituitary adenoma, ncPA). The identification of novel therapeutic targets for human NFPAs depend on a good understanding of the molecular mechanism of NFPAs [5].

Progression in understanding the mechanism of PAs, especially NFPAs, has been achieved over the last several years. According to the reports, germline mutations in $A I P$ or MEN1 genes are associated with young age-onset PAs [6,7]. The $H G F$ and $c-M E T$ genes are frequently expressed in PAs, and their expressions are correlated with phos-phorylated Akt expression [8]. Durán-Prado et al. [9] identified that sst5TMD4, a truncated variant of so- 
matostatin receptor 5, appeared in $85.0 \%$ PAs rather than normal pituitary, and it may play an inhibitory role in PAs that possess poor response to somatostatin analogs. Raf/ MEK/ERK and PI3K/Akt/mTOR signaling pathways are perturbed in NFPAs [10]. As a target of the $S F 1$ gene in gonadotroph cells, CYP11A1 is up-regulated in human GnPA, and Cypllal promotes survival and proliferation of primary cells and cell lines of rat PAs [5]. Rotondi et al. [11] suggested that the gonadotroph phenotype was strongly associated with AIP expression in NFPAs. The AIP level is higher in GnPA than that in ncPA, and both AIP and cyclinD1 levels are high in most NFPAs. The AIP level correlates with follicle-stimulating hormone $\beta$ (FSH $\beta$ ) and cyclinD1 levels in GnPA. However, AIP is not involved in the aggressiveness of NFPAs [11]. Recently, CCNB1 was found to mediate the proliferation-inhibiting role of miR-410, a small non-coding RNA, in GnPA [12]. Additionally, Chesnokova et al. [13] have identified that human pituitary tumors originated from gonadotroph cells express abundant FOXL2, and both FOXL2 and PTTG promote cluster- ing expression and secretion from gonadotroph cells, thus restraining the proliferation of pituitary cells.

Along with the development of microarray, transcriptome analysis has been widely utilized in understanding tumor mechanism. Based on the gene expression microarray dataset GSE26966, Michaelis et al. [14] identified that GADD45 $\beta$, a downstream effector of $\mathrm{p} 53$, is a tumor suppressor in gonadotroph tumor. Its overexpression in mouse gonadotroph cells blocks cell proliferation and promotes apoptosis [14]. Based on the same dataset, Cai et al. [15] identified the coexpressed and altered genes involved in gonadotroph tumors and suggest that ITGA4, MPP2, DLK1, CDKN2A and ASAP2 might be biomarkers. However, pathways or functions of the altered genes were not studied by Michaelis et al. [14], and the protein-protein interactions (PPIs) between genes were not investigated in the two aforementioned studies [14,15]. In particular, Zhao et al. [16] performed an integrated analysis of five available microarray datasets of various PAs, to detect 3994 differentially expressed genes (DEGs) (including 2043 up- and 1951 down-regulated genes), and conducted a PPI network analysis. However, PPIs of more DEGs are needed to be analyzed, and more potential novel PAs-related genes are still unknown. Moreover, molecular mechanisms underlying the pathogenesis of PAs, particular NFPAs, remain unclear, and it is still essential to comprehensively investigate and annotate the alterations in gene expression profiles. In the present study, NFPAs-related microarray data uploaded by Michaelis et al. [14] were analyzed to identify significant DEGs, study NFPAs-related functions and pathways, construct interaction network, and identify potential novel NFPAs-related genes.

\section{MATERIALS AND METHODS}

Microarray Data. Microarray dataset of gene expression, GSE26966 [14], was downloaded from the Gene Expression Omnibus (http://www.ncbi.nlm.nih.gov/geo/ query/acc.cgi?acc=GSE26966). In this dataset, nine normal human pituitary samples were collected from individuals without an endocrine dysfunction at autopsy 2-18 hours post death, and 14 NFPAs samples were obtained from patients at the time of transsphenoidal surgery after obtaining the patient's or their families' permission [14]. Moreover, the 14 NFPA samples contained 10 human GnPA samples [histological analysis: $>5.0 \%$ staining for $\alpha$-subunit (ASU), follicle-stimulating hormone (FSH) or lutein-izing hormone (LH)] and four ncPA samples (histological analysis: $<5.0 \%$ staining for ASU, FSH or LH) [14]. Clinical characteristics of tumor samples were: male $/$ female $=8 / 6$, mean age $($ years $)=61.4$, invasive $/$ noninvasive $=7 / 7$, and recurrent $/$ non-recurrent $=5 / 9$. Clinical characteristics of normal controls were: male/female $=4 / 5$ and mean age $($ years $)=55.9$ years that had no significant difference in comparison with tumor samples ( $p$ value $=0.39$ ) [14]. Raw microarray data were collected using Affymetrix Human Genome U133 Plus 2.0 Array (http:// www.ncbi.nlm.nih. gov/geo/query/acc.cgi?acc=GPL570) in the previous study [14].

Pre-Treatment and Differential Analyses. Robust multi-array average algorithm in the affy package (from http://www.bioconductor/org/package/release/bioc/html/ affy.html) [17] in $R$ was chosen for background correction, data normalization, and calculation of expression values. T-test in package simpleaffy [18] was performed, and fold change (FC) values were determined. Then, $p$ values were corrected using the Bonferroni method, and corrected $p$ value $<0.05$ and $\left[\log _{2} \mathrm{FC}\right]>2$ were set as the cut-off to identify DEGs. Thereafter, package Pheatmap (https:// cran.r-project/org/web/packages/pheatmap/index. html) [19] in $R$ was utilized to cluster genes and samples based on the expression values of DEGs.

Functional and Pathway Enrichment Analyses. Gene Ontology (GO) enrichment and Kyoto Encyclopedia of Genes and Genomes (KEGG) pathway analyses were conducted using package GOstats (http://www.biocon ductor.org/packages/release/bioc/GOstats.html) [20]. The $p$ value $<0.05$ was set as the threshold. User data mapping 
module in the KEGG database (http://www.kegg/jp) was utilized to visualize the significantly enriched pathways.

Construction of Protein-Protein Interaction Network. For all of the identified DEGs, a PPI network was constructed with information from a well-known online server, Search Tool for the Retrieval of Interacting Genes/ Proteins version 10 (STRING v10) (http://string-db.org) [21]. Only the PPIs with a confidence score of $>0.4$ were defined as significant PPIs, which were then utilized to construct the PPI network. The network was visualized using software Cytoscape version 2.8 (http://www.cyto scape.org) [22], and node degrees were determined.

Potential Novel Non-Functioning Pituitary Adenoma-Related Genes and Sub-Network. In order to find potential novel disease genes, known genes implicated in pituitary tumorigenesis were obtained from the Comparative Toxicogenomics Database (CTD) (the most recently released version was up-dated on February 9 2016, http:// ctdbase.org/) [23]. Afterwards, the appearance of these known genes were checked in the PPI network to see whether the known genes were DEGs. Common genes, namely, the overlapped genes, were marked in the PPI network. Other DEGs were defined as potential novel NFPAs-related genes, as they were significantly altered in NFPA specimens and interacted with known disease genes. Furthermore, the top 10 significant DEGs, and DEGs directly interacting with the top DEGs, were extracted to construct a sub-network.

\section{RESULTS}

Differentially Expressed Genes and Clusters. A total of 604 DEGs were acquired between NFPAs and controls, involving 177 up- and 427 down-regulated genes. The top 10 up-regulated genes and top 10 down-regulated genes are shown in Table 1. The 604 DEGs and 23 samples were clustered, and DEGs could well differentiate the disease samples from the healthy controls (Figure 1).

Functions and Pathways. The GO enrichment analysis and KEGG pathway analysis were performed to reveal the key biological functions altered in NFPAs. As shown in Table 2, 12 pathways were significantly enriched, which

Table 1. The top 10 up-regulated genes and top 10 down-regulated genes.

\begin{tabular}{|c|c|c|c|}
\hline Genes & $\log _{2} F C$ & $\begin{array}{c}\text { Corrected } \\
p \text { Value }\end{array}$ & Gene Title \\
\hline \multicolumn{4}{|l|}{ Up-regulated } \\
\hline$S S B P 2$ & 2.04 & $1.43 \mathrm{E}-10$ & single-stranded DNA binding protein 2 \\
\hline $\mathrm{CDH10}$ & 2.68 & $1.43 \mathrm{E}-10$ & cadherin 10, type 2 (T2-cadedrin) \\
\hline FAM171A1 & 2.18 & $2.45 \mathrm{E}-10$ & family with sequency similarity 171 , member A1 \\
\hline EFNB3 & 2.05 & $8.76 \mathrm{E}-10$ & ephrin-B3 \\
\hline PCYT1B & 2.16 & $9.13 \mathrm{E}-10$ & phosphate cytidylytransferase 1 , choline, $\beta$ \\
\hline$R N F 157$ & 2.26 & $1.16 \mathrm{E}-09$ & ring finger protein 157 \\
\hline$C D K 18$ & 2.46 & $1.57 \mathrm{E}-09$ & cyclin-dependent kinase 18 \\
\hline LRFN5 & 3.63 & $2.01 \mathrm{E}-09$ & leucine rich repeat and fibronectin type III domain containing 5 \\
\hline CACNA2D4 & 4.11 & $2.92 \mathrm{E}-09$ & calcium channel, voltage-dependent, a2/ $\delta$ subunit 4 \\
\hline PPARGC1B & 2.97 & 5.94E-09 & peroxisome proliferator-activated receptor $\gamma$, coactivator $1 \beta$ \\
\hline \multicolumn{4}{|c|}{ Down-regulated } \\
\hline GH1 & -9.74 & $1.49 \mathrm{E}-21$ & growth hormone 1 \\
\hline CSH1 & -8.67 & $3.69 \mathrm{E}-15$ & chorionic somatomammotropin hormone 1 (placental lactogen) \\
\hline$D L K 1$ & -9.33 & $4.16 \mathrm{E}-15$ & $\delta$-like 1 homolog (Drosophila) \\
\hline $\mathrm{CSH} 2$ & -9.17 & $3.14 \mathrm{E}-13$ & chorionic somatomammotropin hormone 2 \\
\hline$H I P 1 R$ & -2.16 & $5.43 \mathrm{E}-12$ & huntingtin interacting protein 1 related \\
\hline$C D K N 2 A$ & -2.33 & 4.74E-11 & cyclin-dependent kinase inhibitor 2A (melanoma, p16, inhibits CDK4) \\
\hline$M G P$ & -2.86 & $8.06 \mathrm{E}-11$ & matrix Gla protein \\
\hline KCNJ6 & -3.79 & $9.50 \mathrm{E}-11$ & potassium inwardly-rectifying channel, subfamily J, member 6 \\
\hline SPRY4 & -2.32 & $1.03 \mathrm{E}-10$ & sprouty homolog 4 (Drosophila) \\
\hline MEG3 & -5.72 & $1.60 \mathrm{E}-10$ & maternally expressed 3 (non-protein coding) \\
\hline
\end{tabular}

Corrected $p$ value $<0.05$ and $\left[\log _{2} \mathrm{FC}\right.$ (fold change) $]>2$ were set as the cut-off to identify differentially expressed genes. 
Table 2. Significantly enriched terms.

\begin{tabular}{|c|c|c|c|c|c|}
\hline Category & Term ID & $\begin{array}{l}\text { Corrected } \\
p \text { Value }\end{array}$ & $\begin{array}{l}\text { Number } \\
\text { of DEGs }\end{array}$ & $\begin{array}{l}\text { Number } \\
\text { of Genes }\end{array}$ & Term \\
\hline \multirow[t]{12}{*}{ KEGG } & K04610 & $3.18 \mathrm{E}-04$ & 10 & 69 & complement and coagulation cascades \\
\hline & K04512 & 3.97E-04 & 11 & 84 & extracellular matrix-receptor interaction \\
\hline & K04010 & $4.55 \mathrm{E}-03$ & 20 & 271 & MAPK signaling pathway \\
\hline & K04115 & 5.32E-02 & 8 & 69 & p53 signaling pathway \\
\hline & K00350 & $6.79 \mathrm{E}-03$ & 9 & 87 & transforming growth factor $\beta$ signaling pathway \\
\hline & K04630 & $7.62 \mathrm{E}-03$ & 13 & 155 & Jak-STAT signaling pathway \\
\hline & K04080 & $1.14 \mathrm{E}-02$ & 18 & 256 & neuroactive ligand-receptor interaction \\
\hline & K04510 & $1.27 \mathrm{E}-02$ & 15 & 202 & focal adhesion \\
\hline & K05218 & $2.08 \mathrm{E}-02$ & 7 & 71 & Melanoma \\
\hline & K05412 & $2.90 \mathrm{E}-02$ & 7 & 76 & arrhythmogenic right ventricular cardiomopathy \\
\hline & K05210 & 4.63E-02 & 7 & 84 & colorectal cancer \\
\hline & K04920 & $4.70 \mathrm{E}-02$ & 6 & 67 & adipocytokine signaling pathway \\
\hline GO BP & GO:0032501 & $2.26 \mathrm{E}-24$ & 266 & 4974 & multicellular organismal process \\
\hline \multirow[t]{9}{*}{ (top 10) } & GO:0010243 & $6.92 \mathrm{E}-13$ & 57 & 596 & response to organic nitrogen \\
\hline & GO:0007275 & 4.19E-12 & 112 & 2080 & multicellular organismal development \\
\hline & GO:0048583 & $2.00 \mathrm{E}-10$ & 99 & 1624 & regulation of response to stimulus \\
\hline & GO:0023051 & 4.23E-10 & 110 & 1866 & regulation of signaling \\
\hline & GO:0010646 & $5.12 \mathrm{E}-10$ & 110 & 1872 & regulation of cell communication \\
\hline & GO:0048812 & $1.38 \mathrm{E}-09$ & 49 & 571 & neuron projection morphogenesis \\
\hline & GO:0048667 & 3.03E-09 & 48 & 566 & cell morphogenesis involved in neuron differentiation \\
\hline & GO:0007243 & 3.09E-09 & 64 & 879 & intracellular protein kinase cascade \\
\hline & GO:0022008 & $6.39 \mathrm{E}-09$ & 63 & 900 & neurogenesis \\
\hline GO CC & GO:0005576 & $1.31 \mathrm{E}-14$ & 132 & 2164 & extracellular region \\
\hline \multirow[t]{9}{*}{ (top 10) } & GO:0005615 & 1.19-E-09 & 61 & 848 & extracellular space \\
\hline & GO:0005587 & $1.37 \mathrm{E}-05$ & 4 & 6 & collagen type IV \\
\hline & GO:0005581 & $1.90 \mathrm{E}-05$ & 12 & 88 & collagen \\
\hline & GO:0043005 & 5.12E-05 & 39 & 634 & neuron projection \\
\hline & GO:0005578 & $5.72 \mathrm{E}-05$ & 18 & 204 & proteinaceous extracellular matrix \\
\hline & GO:0031012 & $8.74 \mathrm{E}-05$ & 9 & 62 & extracellular matrix \\
\hline & GO:0016323 & $1.39 \mathrm{E}-04$ & 15 & 158 & basolateral plasma membrane \\
\hline & GO:0005887 & $5.55 \mathrm{E}-04$ & 59 & 1216 & integral to plasma membrane \\
\hline & GO:0005584 & 9.84E-04 & 2 & 2 & collagen type I \\
\hline GO MF & GO:0005201 & $2.40 \mathrm{E}-10$ & 17 & 78 & extracellular matrix structural constituent \\
\hline \multirow[t]{9}{*}{ (top 10) } & GO:0008201 & $1.20 \mathrm{E}-07$ & 18 & 129 & heparin binding \\
\hline & GO:0097367 & $1.60 \mathrm{E}-07$ & 22 & 191 & carbohydrate derivative binding \\
\hline & GO:0042803 & 5.71E-06 & 38 & 553 & protein homodimerization binding \\
\hline & GO:0005179 & $9.11 \mathrm{E}-11$ & 14 & 110 & hormone activity \\
\hline & GO:0000981 & $1.75 \mathrm{E}-05$ & 22 & 253 & $\begin{array}{l}\text { sequence specific DNA binding RNA polymerase II } \\
\text { transcription factor activity }\end{array}$ \\
\hline & GO:0001077 & $4.36 \mathrm{E}-05$ & 10 & 67 & $\begin{array}{l}\text { RNA polymerase II core promoter proximal region } \\
\text { sequence-specific DNA binding transcription factor activity } \\
\text { involved in positive regulation of transcription }\end{array}$ \\
\hline & GO:0019199 & $5.05 \mathrm{E}-05$ & 11 & 82 & transmembrane receptor protein kinase activity \\
\hline & GO:0005102 & $1.92 \mathrm{E}-04$ & 55 & 1093 & receptor binding \\
\hline & GO:0048407 & $2.70 \mathrm{E}-04$ & 4 & 11 & platelet-derived growth factor binding \\
\hline
\end{tabular}

ID: identifier; DEGs: differentially expressed genes; KEGG: Kyoto Encyclopedia of Genes and Genomes; GO: gene ontology;

BP: biological process; CC: cellular component; MF: molecular functions. 


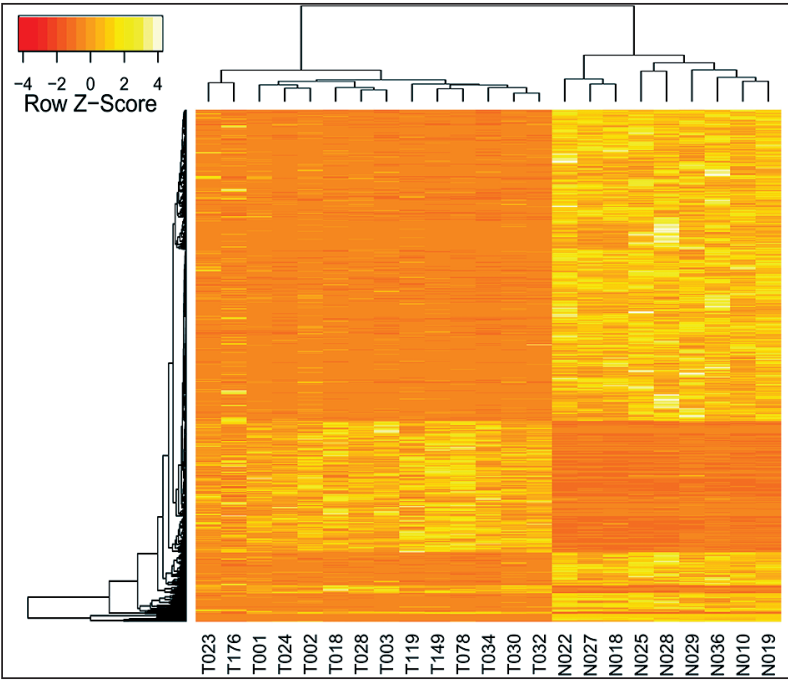

Figure 1. Cluster analysis of DEGs. DEGs: differentially expressed genes; T: tumor samples; $\mathrm{N}$ : healthy normal samples. Cluster analysis was performed both at gene level (vertical) and sample level (horizontal).

were mainly associated with signaling pathway and receptor interaction. In GO enrichment analysis, DEGs were significantly enriched in 1037 biological process terms mainly about cell communication and signaling, 65 cellular component terms mainly related with an extracellular matrix (ECM), plasma membrane, and collagen, as well as 186 molecular function terms mainly associated with transcription factor activity and receptor binding (Table 2 ). In order to better understand the positions of DEGs in pathways and their roles in the development of NFPAs, we visualized four significant pathways that had been reported to participate in the pathogenesis of NFPAs or PAs, including MAPK signaling pathway [10] (Figure 2), p53 signaling pathway [24] (Figure 3), transforming growth factor $\beta$ (TGF $\beta$ ), signaling pathway [25] (Figure 4), and Jak-STAT signaling pathway [8] (Figure 5).

Protein-Protein Interaction Network of Differentially Expressed Genes. For the 604 DEGs, the PPI network was constructed using information from STRING v10 (Figure 6). The whole network consisted of 115 upregulated DEGs, 305 down-regulated DEGs and 1379 PPIs (Figure 6).

Potential Novel Non-Functioning Pituitary Adenoma-Related Genes and Sub-Network. Known disease genes were obtained from the CTD database (http://ctd

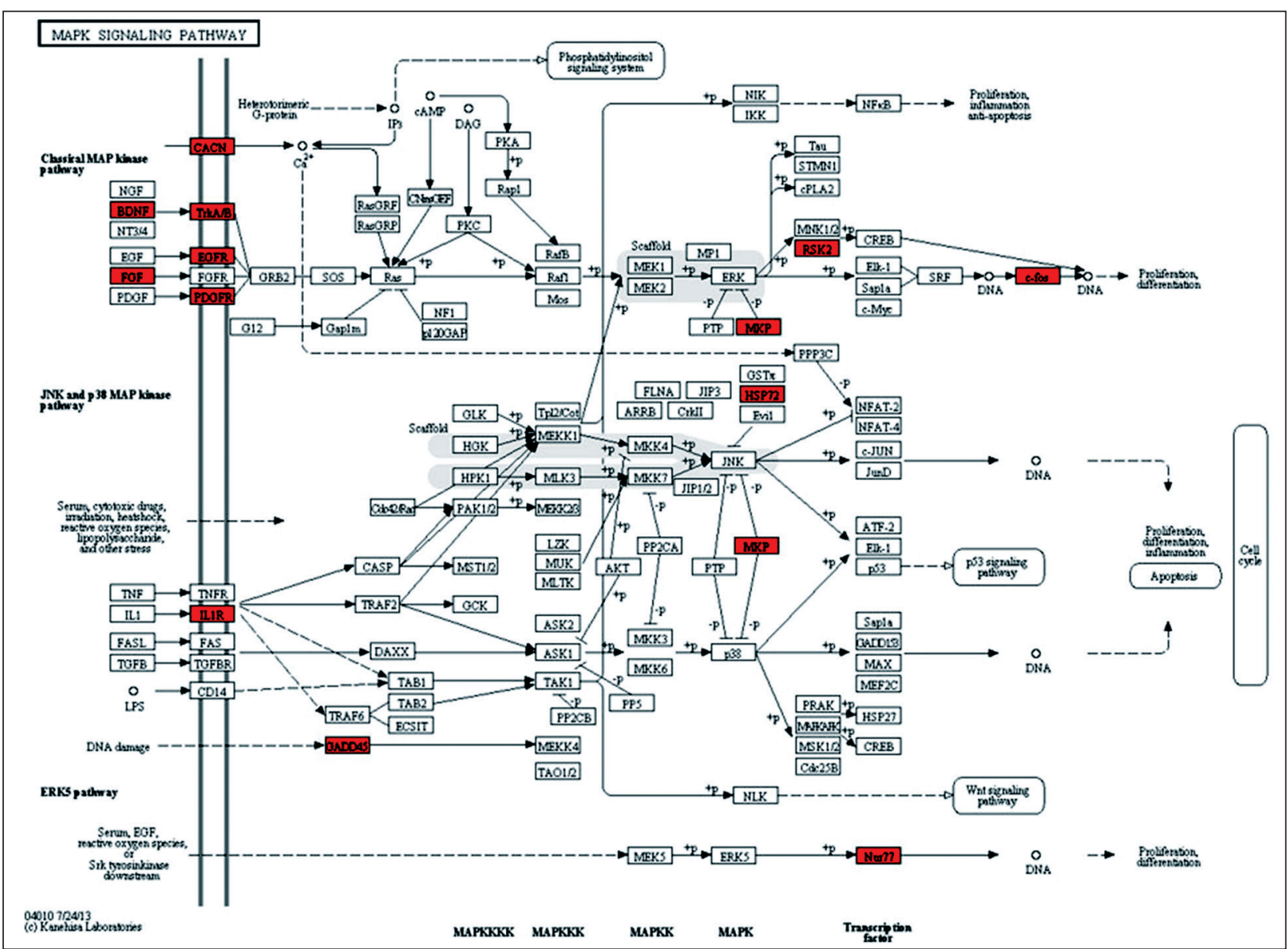

Figure 2. The MAPK signaling pathway. Genes down-regulated in NFPAs are shown in green, while up-regulated genes are in red. 


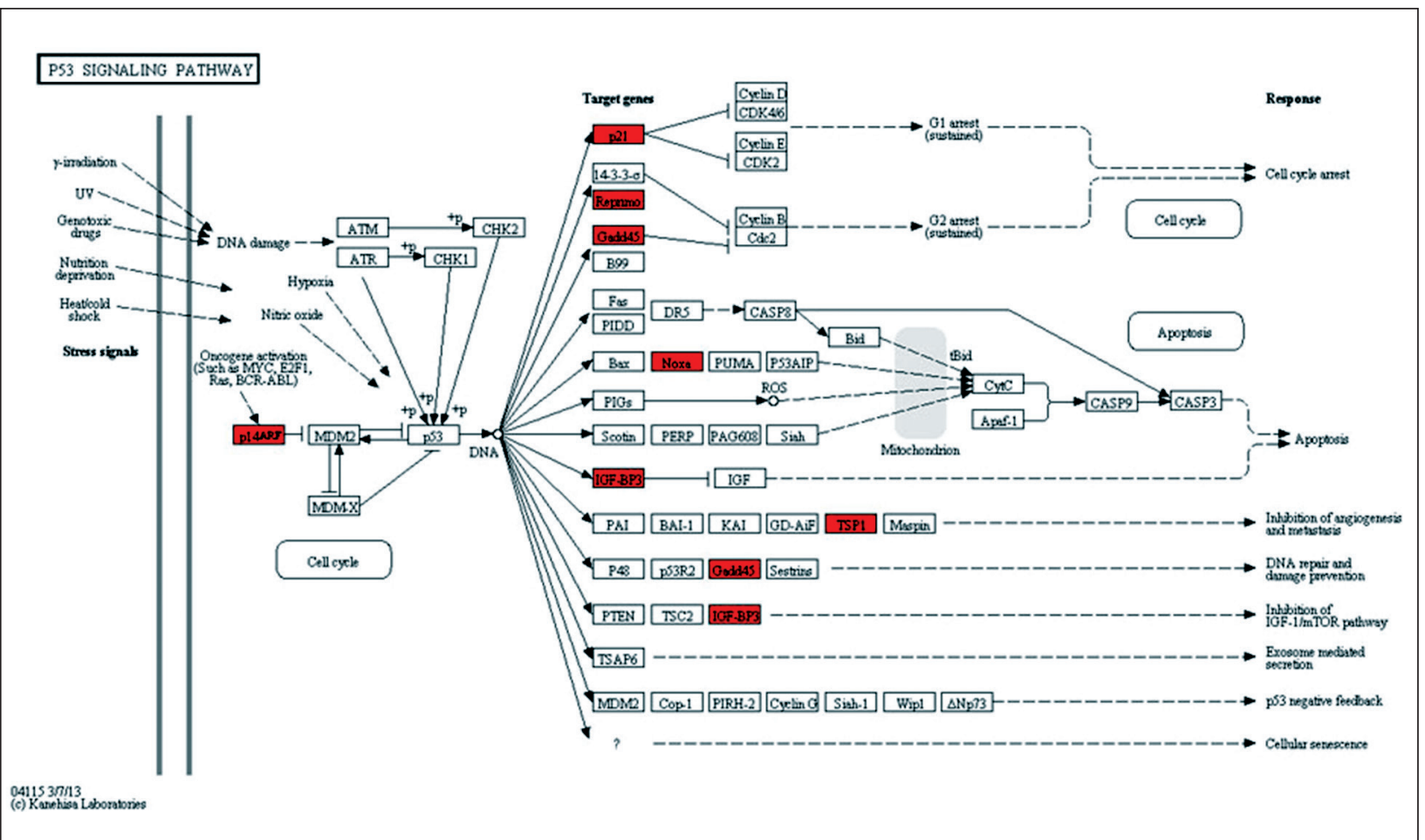

Figure 3. The p53 signaling pathway. Genes down-regulated in NFPAs are shown in green, while up-regulated genes are in red.

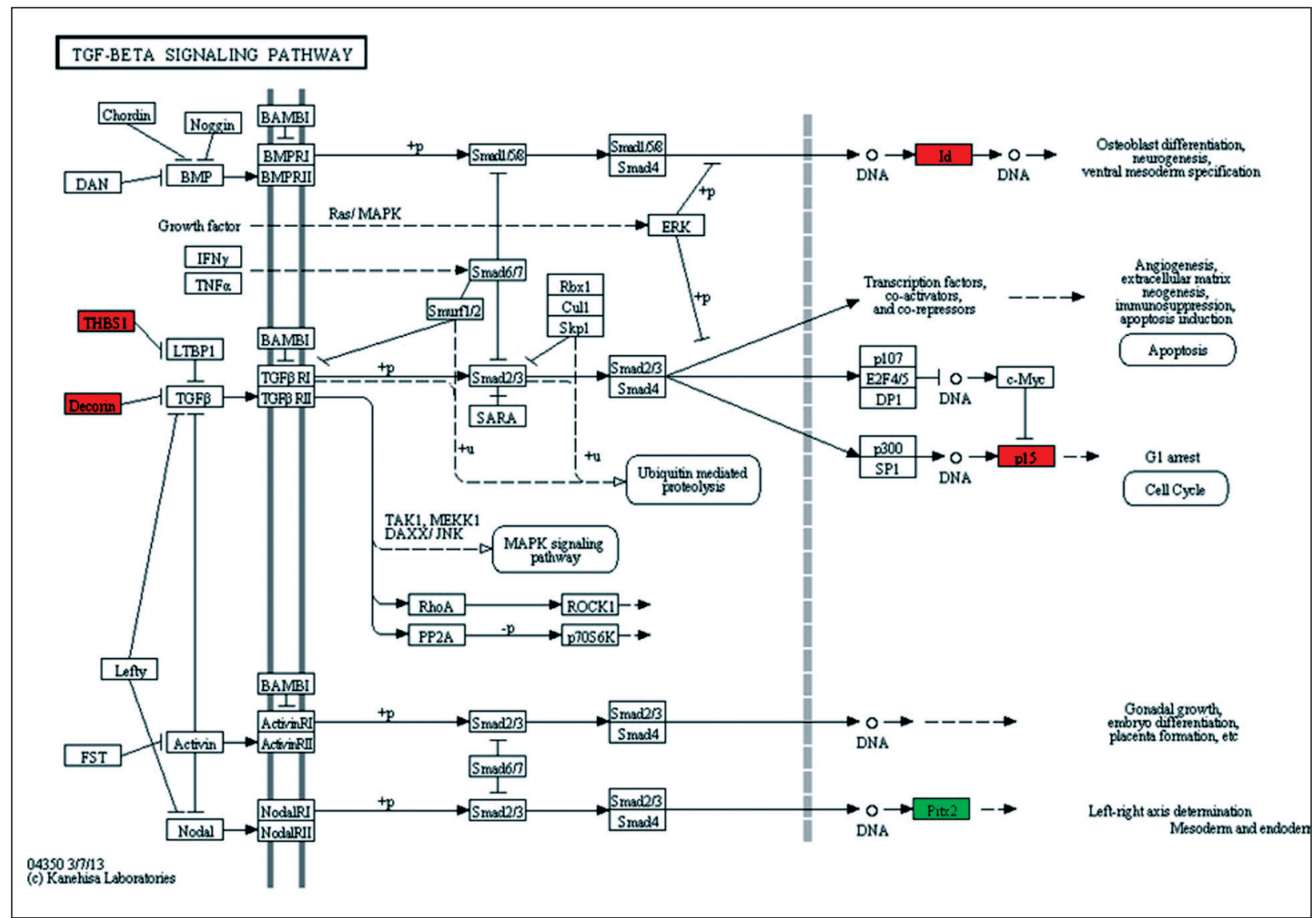

Figure 4. The TGF $\beta$ signaling pathway. Genes down-regulated in NFPAs are shown in green, while up-regulated genes are in red. 


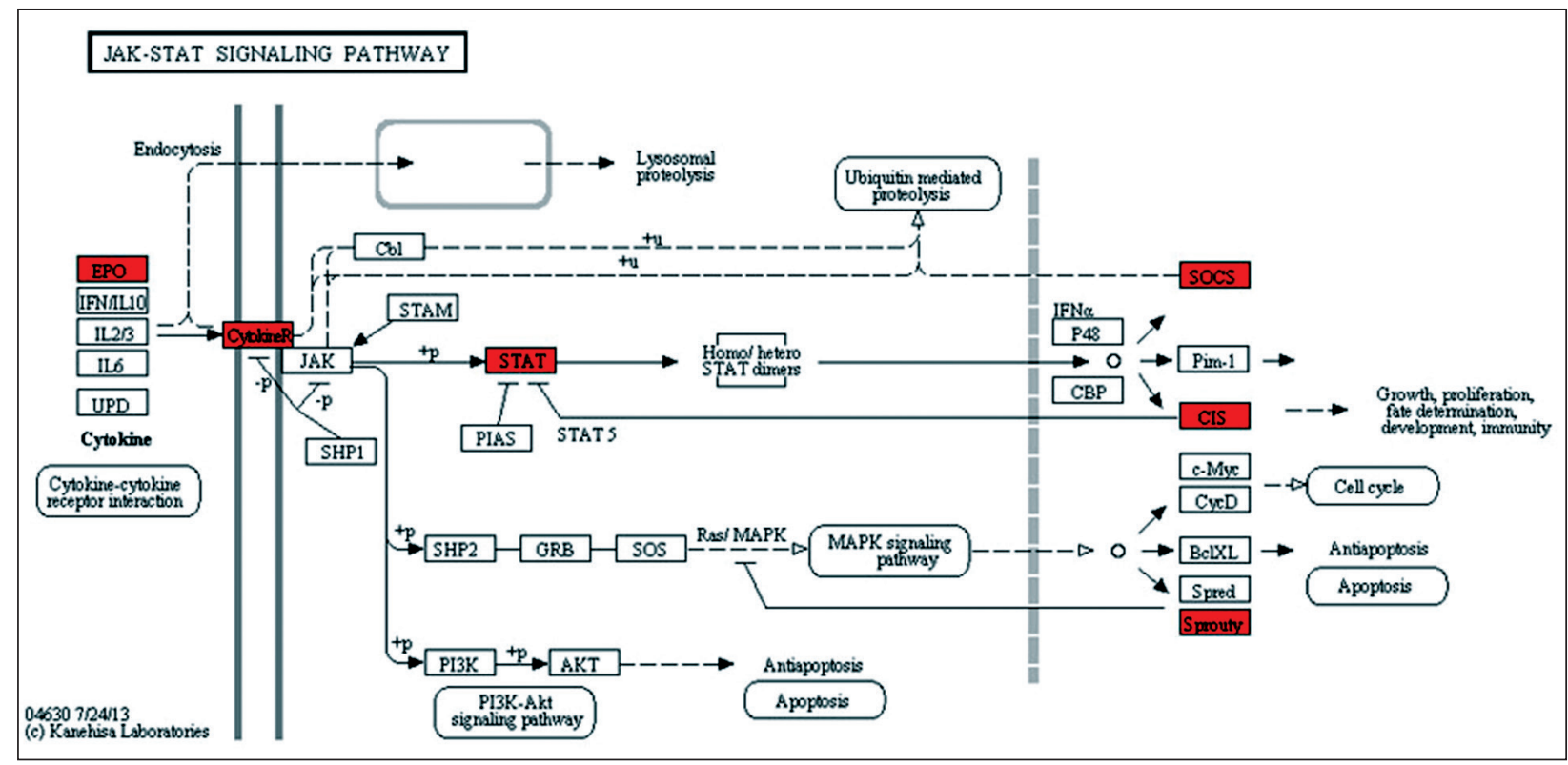

Figure 5. Jak-STAT signaling pathway. Genes down-regulated in NFPAs are shown in green, while up-regulated genes are in red.

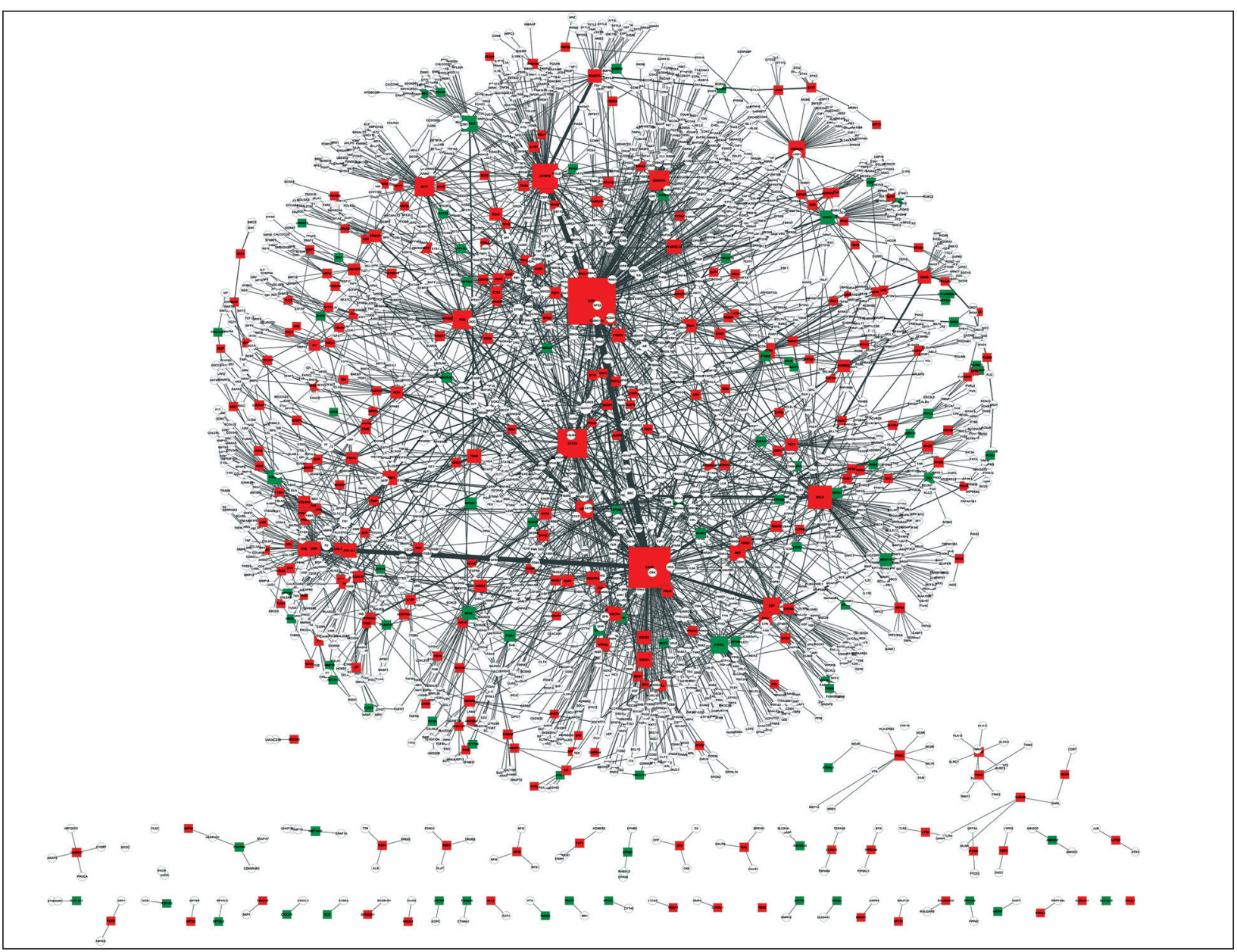

Figure 6. The whole PPI network of DEGs. Red nodes represent the genes up-regulated in NFPAs, and green nodes represent the genes down-regulated in NFPAs. Circle nodes stand for known disease genes, whereas triangle nodes stand for potential novel disease genes. Node size positively correlates with node degree, namely, the number of neighbors. PPI: protein-protein interaction; DEGs: differentially expressed genes; NFPAs: non-functioning pituitary adenomas. 


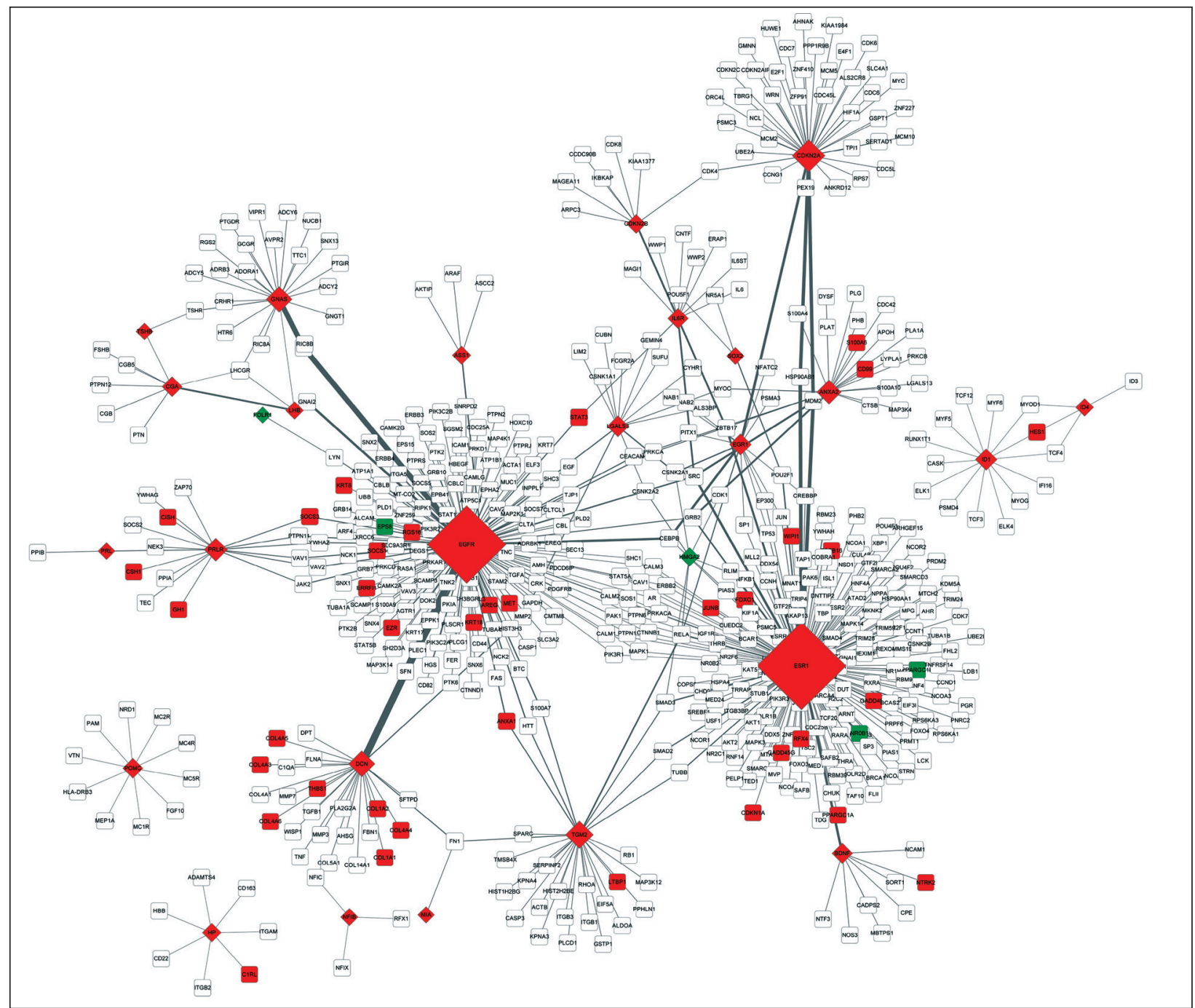

Figure 7. The PPI sub-network containing the top 10 DEGs. Red nodes represent the genes up-regulated in NFPAs, and green nodes represent the genes down-regulated in NFPAs. Circle nodes stand for known disease genes, whereas triangle nodes stand for potential novel disease genes. Node size positively correlates with node degree, namely, the number of neighbors. PPI: protein-protein interaction; DEGs: differentially expressed genes; NFPAs: non-functioning pituitary adenomas.

base.org/) and compared with the DEGs in the PPI network. Consequently, 99 up- and 288 down-regulated DEGs were known disease genes, e.g. EGFR (epidermal growth factor receptor, degree $=63$ ) $[10,26-28]$ and ESRI (estrogen receptor 1 , degree $=48$ ) [29] (Figure 6). In contrast, 16 up- and 17 down-regulated DEGs were potential novel NFPA-related genes, e.g. COL4A5 (collagen type IV $\alpha 5$, degree $=17), L H X 3$ (LIM homeobox protein 3, degree = 11), MSN (moesin, degree =11) and GHSR (growth hormone secretagogue receptor, degree $=10$ ) (Figure 6). Moreover, COL4A5 interacted with known NFPA-related genes such as EGFR, $L H X 3$ interacted with known NFPAsrelated genes like $P R L$ (Prolactin), and $M S N$ interacted with known NFPA-related genes such as EGFR. Among the top 10 up-regulated genes and top 10 down-regulated genes, only 12 DEGs interacted with other DEGs [e.g. CD$K N 2 A$ (cyclin-dependent kinase inhibitor 2A)-IDH1 (isocitrate dehydrogenase 1)], and all 12 DEGs were known disease genes [e.g. $D L K 1$ ( $\delta$-like 1 homologue)] (Figure 7). In addition, potential NFPA-related gene GHSR interacted with the top DEG GH1 (growth hormone 1).

\section{DISCUSSION}

Non-functioning pituitary adenomas comprise about $34.0 \%$ of pituitary tumors, while their molecular mechanism is still incompletely understood [5]. In the current study, we comprehensively analyzed the gene expression 
profile of NFPAs and healthy pituitary glands. As a result, 604 DEGs were identified between NFPAs and controls, including 177 up- and 427 down-regulated genes, which were much less than those identified by Michaelis et al. [14]. However, in the current study, we analyzed the same microarray data using different software, algorithms, and analysis criteria (corrected $p$ value $<0.05$ and $\left[\log _{2} \mathrm{FC}\right]>2$ ) in order to focus on the DEGs that were more significant.

In the current study, mean FC of the up-regulated genes was 6.6, and mean FC of the down-regulated genes was -19.2 , which were different from those in the previous study by Michaelis et al. [14] (4.5 and-32.2, respectively). The differences of mean FC values might be caused by the different DEG sets in the two studies [14]. The major DEGs found by Michaelis et al. [14] had similar expression change patterns in the current study, e.g. for the PLAGL1, CDKN1A, RPRM, PMAIP1, MDM2, GADD45A, GAD$D 45 B$ and $G A D D 45 G$ genes.

Of the top DEGs, DLK1, GH1, CDKN2A and MEG3 were significantly down-regulated in NFPAs in comparison with normal pituitary glands in this study. According to the report, the $M E G 3$ and $D L K 1-M E G 3$ locus are silenced in human NFPAs of gonadotroph origin, and DLK1-MEG3 locus plays a tumor suppressor role in NFPAs [30]. Based on proteome data and microarray data or reverse transcription quantitative real-time polymerase chain reaction analysis, Moreno et al. [31] found that DLK1, GH1 and PRL are down-regulated in NFPAs when compared with normal pituitary glands, whereas $I D H 1$ is significantly up-regulated. The CDKN2A and DLK1 are considered as biomarkers of gonadotroph tumors by Cai et al. [15], and gene silencing mediated by hypermethylation of the $\mathrm{CpG}$ island within exon 1 in CDKN2A is associated with NFPAs [32]. As clearly shown in Figure 7, the expression change patterns of known disease genes DLK1, GH1, PRL, CDKN2A and $I D H 1$, were consistent with the aforementioned studies [30-32], demonstrating the high accuracy of our results.

Expressions of EGFR in NFPAs varied in different studies [10,26-28]. In the current study, EGFR showed low expression in NFPAs (Figure 7), and it interacted with known disease gene $C D K N 2 A$, indicating that low expression of $E G F R$ might be associated with NFPAs. We also found that $C D K N 2 A$ was a top DEG, and it interacted with 22 DEGs in the whole PPI network and most DEGs in the PPI sub-network, suggesting that $C D K N 2 A$ might play a crucial role in the progression of NFPAs.

Furthermore, potential novel genes were identified (Figure 6), especially COL4A5, LHX3, MSN and GHSR. The role of these genes in NFPAs has not been investigated by previous studies. According to the report, mRNA level of GHSR in NFPAs is lower than that in growth hormoneproducing PAs [33]. In the present study, COL4A5, LHX3, $M S N$ and GHSR were significantly down-regulated in NFPAs in comparison with normal controls, and they interacted with known NFPA-related genes such as EGFR, $P R L$, and GH1. These results indicated that COL4A5, LHX3, $M S N$ and GHSR might participate in the initiation and progression of NFPAs via interaction with EGFR, PRL and $G H 1$, respectively.

We found DEGs were significantly enriched in the p53 (Figure 3) and Jak-STAT signaling pathways (Figure 5), which had been reported to take part in PAs pathogenesis $[8,24]$. The p53 signaling pathway is involved in biological processes such as cell cycle arrest, apoptosis, senescence, DNA repair and changes in metabolism. Expression level of $\mathrm{p} 53$ correlates with the proliferative state of PAs [24]. The Jak-STAT pathway is an important downstream pathway for growth factor receptors and cytokine receptors, and it is involved in the regulation of cell proliferation and survival $[34,35]$. As all of the DEGs mapped on these pathways were remarkably down-regulated in NFPAs, p53 and Jak-STAT signaling pathways might play roles in the progression of NFPAs.

In addition, DEGs were significantly enriched in GO terms mainly about cell communication, signaling, ECM, plasma membrane, collagen, transcription factor activity and receptor binding (Table 2). The ECM, plasma membrane, and receptor binding are the basis of cell communication and signaling between pituitary cells, which play crucial roles in the development and invasion of PAs [36, 37]. As DEGs mapped on these GO terms were remarkably dysregulated in NFPAs, cell communication and signaling might contribute to the progression of NFPAs.

In conclusion, a number of genes (e.g. COL4A5, $L H X 3, M S N$ and GHSR) identified in this study, might be potential novel NFPA-related genes. Furthermore, cell communication and signaling pathways (e.g. p53 and JakSTAT) might be implicated in the pathogenesis of NFPAs. Currently, no effective medical therapies are available for NFPAs, due to their unclear mechanism. Although further validation is required, our findings might provide information to guide future researchers and even benefit the development of medical therapy for NFPAs.

Declaration of Interest. This study was supported by Natural Science Fund (grant number: 20150101193JC). The authors report no conflicts of interest. The authors alone are responsible for the content and writing of this article. 


\section{REFERENCES}

1. Gruppetta M, Mercieca C, Vassallo J. Prevalence and incidence of pituitary adenomas: A population based study in Malta. Pituitary. 2013; 16(4): 545-553.

2. Karavitaki N. Prevalence and incidence of pituitary adenomas. Ann Endocrinol (Paris). 2012; 73(2): 79-80.

3. Pereira AM, Biermasz NR. Treatment of nonfunctioning pituitary adenomas: What were the contributions of the last 10 years? A critical view. Ann Endocrinol (Paris). 2012; 73(2): 111-116.

4. Chaidarun SS, Klibanski A. Gonadotropinomas. Semin Reprod Med. 2002; 20(4): 339-348.

5. Lee M, Marinoni I, Irmler M, Psaras T, Honegger $\mathrm{JB}$, Beschorner R, et al. Transcriptome analysis of MENX-associated rat pituitary adenomas identifies novel molecular mechanisms involved in the pathogenesis of human pituitary gonadotroph adenomas. Acta Neuropathol. 2013; 126(1): 137-150.

6. Beckers A, Aaltonen LA, Daly AF, Karhu A. Familial isolated pituitary adenomas (FIPA) and the pituitary adenoma predisposition due to mutations in the aryl hydrocarbon receptor interacting protein (AIP) gene. Endocr Rev. 2013; 34(2): 239-277.

7. Stratakis CA, Tichomirowa MA, Boikos S, Azevedo MF, Lodish M, Martari M, et al. The role of germline AIP, MEN1, PRKAR1A, CDKN1B and CDKN2C mutations in causing pituitary adenomas in a large cohort of children, adolescents, and patients with genetic syndromes. Clin Genet. 2010; 78(5): 457-463.

8. Trovato M, Torre ML, Ragonese M, Simone A, Scarfi R, Barresi V, et al. HGF/c-met system targeting PI3K/ AKT and STAT3/phosphorylated-STAT3 pathways in pituitary adenomas: An immunohistochemical characterization in view of targeted therapies. Endocrine. 2013; 44(3): 735-743.

9. Duran-Prado M, Saveanu A, Luque RM, Gahete MD, Gracia-Navarro F, Jaquet $\mathrm{P}$, et al. A potential inhibitory role for the new truncated variant of somatostatin receptor 5, sst5TMD4, in pituitary adenomas poorly responsive to somatostatin analogs. J Clin Endocrinol Metab. 2010; 95(5): 2497-2502.

10. Rubinfeld H, Shimon I. PI3K/Akt/mTOR and Raf/ $\mathrm{MEK} / \mathrm{ERK}$ signaling pathways perturbations in nonfunctioning pituitary adenomas. Endocrine. 2012; 42(2): 285-291.

11. Rotondi S, Oliva MA, Esposito V, Ventura L, Giangaspero F, Alesse E, et al. AIP expression in non-functioning pituitary adenomas is strongly associated with the gonadotroph phenotype but not with tumour aggressiveness. Endocrine Abstracts. 2014; 35: P835. (hppt:// www.endocrine-abstracts. org/ea/0035/ea0035P835/ htm).

12. Mussnich P, Raverot G, Jaffrain-Rea ML, Fraggetta F, Wierinckx A, Trouillas J, et al. Downregulation of miR-410 targeting the cyclin B1 gene plays a role in pituitary gonadotroph tumors. Cell Cycle. 2015; 14(16): 2590-2597.

13. Chesnokova V, Zonis S, Wawrowsky K, Tani Y, BenShlomo A, Ljubimov V, et al. Clusterin and FOXL2 act concordantly to regulate pituitary gonadotroph adenoma growth. Mol Endocrinol. 2012; 26(12): 2092-2103.

14. Michaelis KA, Knox AJ, Xu M, Kiseljak-Vassiliades $\mathrm{K}$, Edwards MG, Geraci M, et al. Identification of growth arrest and DNA-damage-inducible gene beta (GADD45beta) as a novel tumor suppressor in pituitary gonadotrope tumors. Endocrinology. 2011; 152(10): 3603-3613.

15. Cai T, Xiao J, Wang ZF, Liu Q, Wu H, Qiu YZ. Identification of differentially coexpressed genes in gona-dotrope tumors and normal pituitary using bioinformatics methods. Pathol Oncol Res. 2014; 20(2): 375-380.

16. Zhao $\mathrm{P}, \mathrm{Hu} \mathrm{W}$, Wang $\mathrm{H}, \mathrm{Yu} \mathrm{S}, \mathrm{Li} \mathrm{C}$, Bai J, et al. Identification of differentially expressed genes in pituitary adenomas by integrating analysis of microarray data. Int J Endocrinol. 2015; 2015: 164087. doi: 10.1155/2015/ 164087.

17. Gautier L, Cope L, Bolstad BM, Irizarry RA. affyanalysis of Affymetrix GeneChip data at the probe level. Bioinformatics. 2004; 20(3): 307-315.

18. Wilson CL, Miller CJ. Simpleaffy: A BioConductor package for Affymetrix Quality Control and data analysis. Bioinformatics. 2005; 21(18): 3683-3685.

19. Kolde R. Pheatmap: Pretty Heatmaps. R Package Version 0.7. 7. CRAN Repository, 2012.

20. Falcon S, Gentleman R. Using GOstats to test gene lists for GO term association. Bioinformatics. 2007; 23(2): 257-258.

21. Szklarczyk D, Franceschini A, Wyder S, Forslund K, Heller D, Huerta-Cepas J, et al. STRING v10: Protein-protein interaction networks, integrated over the tree of life. Nucleic Acids Res. 2015; 43(Database issue): D447-D452. 
22. Kohl M, Wiese S, Warscheid B. Cytoscape: Software for visualization and analysis of biological networks. Methods Mol Biol. 2011; 696: 291-303.

23. Davis AP, Grondin CJ, Lennon-Hopkins K, SaraceniRichards C, Sciaky D, King BL, et al. The Comparative Toxicogenomics Database's 10th year anniversary: Update 2015. Nucleic Acids Res. 2015; 43(Database issue): D914-D920.

24. Suliman M, Royds J, Cullen D, Timperley W, Powell $\mathrm{T}$, Battersby R, et al. Mdm2 and the $\mathrm{p} 53$ pathway in human pituitary adenomas. Clin Endocrinol (Oxf). 2001; 54(3): 317-325.

25. Butz H, Likó I, Czirják S, Igaz P, Korbonits M, Rácz $\mathrm{K}$, et al. MicroRNA profile indicates downregulation of the TGF $\beta$ pathway in sporadic non-functioning pituitary adenomas. Pituitary. 2011; 14(2): 112-124.

26. Rishi A, Sharma MC, Sarkar C, Jain D, Singh M, Mahapatra AK, et al. A clinicopathological and immuno-histochemical study of clinically non-functioning pituitary adenomas: A single institutional experience. Neurol India. 2010; 58(3): 418-423.

27. Chaidarun SS, Eggo MC, Sheppard MC, Stewart PM. Expression of epidermal growth factor (EGF), its receptor, and related oncoprotein (erbB-2) in human pituitary tumors and response to EGF in vitro. Endocrinology. 1994; 135(5): 2012-2021.

28. Onguru O, Scheithauer BW, Kovacs K, Vidal S, Jin $\mathrm{L}$, Zhang S, et al. Analysis of epidermal growth factor receptor and activated epidermal growth factor receptor expression in pituitary adenomas and carcinomas. Mod Pathol. 2004; 17(7): 772-780.

29. Chaidarun SS, Klibanski A, Alexander JM. Tumorspecific expression of alternatively spliced estrogen receptor messenger ribonucleic acid variants in human pituitary adenomas. J Clin Endocrinol Metab. 1997; 82(4): 1058-1065.
30. Cheunsuchon P, Zhou Y, Zhang X, Lee H, Chen W, Nakayama Y, et al. Silencing of the imprinted DLK1-MEG3 locus in human clinically nonfunctioning pituitary adenomas. Am J Pathol. 2011; 179(4): 2120-2130.

31. Moreno CS, Evans CO, Zhan X, Okor M, Desiderio DM, Oyesiku NM. Novel molecular signaling and classification of human clinically nonfunctional pituitary adenomas identified by gene expression profiling and proteomic analyses. Cancer Res. 2005; 65(22): 10214-10222.

32. Simpson DJ, Bicknell JE, McNicol AM, Clayton RN, Farrell WE. Hypermethylation of the p16/CDKN2A/ MTSI gene and loss of protein expression is associated with nonfunctional pituitary adenomas but not somatotrophinomas. Genes Chromosomes Cancer. 1999; 24(4): 328-336.

33. Kim K, Arai K, Sanno N, Osamura RY, Teramoto A, Shibasaki T. Ghrelin and growth hormone $(\mathrm{GH})$ secre-tagogue receptor (GHSR) mRNA expression in human pituitary adenomas. Clin Endocrinol (Oxf). 2001; 54(6): 759-768.

34. Heim MH. The Jak-STAT pathway: Cytokine signalling from the receptor to the nucleus. J Recept Sig Transd. 1999; 19(1-4): 75-120.

35. Schindler CW. Series introduction: JAK-STAT signaling in human disease. J Clin Invest. 2002; 109(9): 1133-1137.

36. Gong J, Zhao Y, Abdel-Fattah R, Amos S, Xiao A, Lopes MBS, et al. Matrix metalloproteinase-9, a potential biological marker in invasive pituitary adenomas. Pituitary. 2008; 11(1): 37-48.

37. Paez-Pereda M, Kuchenbauer F, Arzt E, Stalla G. Regulation of pituitary hormones and cell proliferation by components of the extracellular matrix. Braz J Med Biol Res. 2005; 38(10): 1487-1494. 
